\title{
Decreased Identification of Vesicoureteral Reflux: A Cautionary Tale
}

\author{
Aslam Hyder Qureshi, ${ }^{1,2}$, Oluwaseun Ajayi ${ }^{3}$, Andrew Lawrence Schwaderer ${ }^{4}$ \\ and David S. Hains ${ }^{1,2 *}$
}

\begin{abstract}
'Innate Immunity Translational Research Center, Children Foundation Research Institute, Le Bonheur Children's Hospital, Memphis, TN, United States, ${ }^{2}$ Department of Pediatrics, University of Tennessee Health Science Center, Memphis, TN, United States, ${ }^{3}$ Biomedical Informatics Core, Children's Foundation Research Institute, Le Bonheur Children's Hospital, Memphis, TN, United States, ${ }^{4}$ Division of Nephrology, Nationwide Children's Hospital, Columbus, OH, United States
\end{abstract}

OPEN ACCESS

Edited by:

Ibrahim F. Shatat, Medical University of South

Carolina and Weill Cornell Medical College, United States; Sidra Medical and Research

Center, Qatar

Reviewed by:

Sherjeel Saulat,

Sindh Institute of Urology and

Transplantation, Pakistan

Howard Trachtman,

New York University,

United States

*Correspondence: David S. Hains dhains@iu.edu

Specialty section: This article was submitted to Pediatric Nephrology, a section of the journal Frontiers in Pediatrics

Received: 30 May 2017

Accepted: 31 July 2017

Published: 11 August 2017

Citation:

Qureshi AH, Ajayi O, Schwaderer AL and Hains DS (2017) Decreased

Identification

of Vesicoureteral Reflux:

A Cautionary Tale.

Front. Pediatr. 5:175.

doi: 10.3389/fped.2017.00175
Aim: To find the trend in patient's visits to our centers for vesicoureteral reflux (VUR). We hypothesize that VUR diagnosis and hence possible nephropathy recognition may be diminishing because of changing practice patterns.

Methods: Data were extracted from electronic medical records for new and follow-up patients aged 0-18 years with ICD-9/10 codes to correspond with VUR, VUR unilateral, VUR bilateral, and VUR with reflux nephropathy, as well as new patients with diagnoses of urinary tract infections (UTI) and pyelonephritis at two major pediatric centers from 2012 to 2015. Figures and statistics to reflect absolute clinic visits and annual trends were created with SPSS 2010. Linear regression was applied.

Results: Annually, Le Bonheur Children's Hospital and Nationwide Children's Hospital experienced an average decrease of 13 and $17 \%$ in total VUR visits, and an average decrease of 22 and $27 \%$ in VUR nephropathy visits, respectively, for each institution. Patient visits for UTIs were reduced an average of $16 \%$ annually in both centers. Linear regression demonstrated that number of patients (patients/year $\pm S E$ ) decreased annually $69 \pm 19(P=0.02), 7 \pm 2(P=0.02)$, and $67 \pm 25(P=0.04)$ for VUR, VUR nephropathy, and UTI, respectively.

Conclusion: We conclude that the decreased number of VUR and VUR nephropathy cases identified in subspecialty clinics (Nephrology/Urology) at two major children's hospitals reflect a possible decreased identification of VUR. This trend may also be due to decreased referral of low grade cases of VUR. We cannot conclude that "undifferentiated UTI" referrals increased concomitantly to account for the decreased VUR as our data reflects a decreased trend in those visits as well. We suggest that clinicians following the American Academy of Pediatrics guidelines ensure that all UTI are accounted for and surveillance is appropriately escalated for recurrent UTI or abnormal imaging results.

Keywords: vesicoureteral reflux, voiding cystourethrogram, renal scarring, pediatrics, renal bladder ultrasound

\section{INTRODUCTION}

Urinary tract infections (UTI) occur in 7\% of children between 0 and 2 years of age (1). Imaging studies after UTI have shown vesicoureteral reflux (VUR) in 30-40\% of children (2). In 1999, the American Academy of Pediatrics (AAP) guidelines recommended renal ultrasound and voiding cystourethrogram (VCUG) after first febrile UTI in children between 2 and 24 months of age to 
evaluate for urinary tract abnormalities. No recommendations were made on continuous antibiotic prophylactic (CAP) therapy (3). The AAP revised guidelines in 2011 recommend renal bladder ultrasound (RBUS) after first febrile UTI and VCUG after second febrile UTI or if RBUS is abnormal (4). Evidence from limited studies at the time did not indicate a role for CAP in preventing scarring (5). However, the risk of renal scarring increases exponentially from $10 \%$ with the second UTI to $60 \%$ following the sixth recurrent UTI (3). Recent evidence from Randomized Intervention for Children with Vesicoureteral Reflux (RIVUR) and Swedish VUR trials suggests definitive role of CAP in certain children with VUR in preventing recurrent UTIs $(6,7)$. In patients with VUR, recurrent UTIs are associated with acquired scarring risk $(8,9)$.

\section{Objective}

We hypothesize that VUR diagnosis and hence possible nephropathy recognition may be diminishing because of changing UTI practice patterns. We collected data to determine the trends of UTI, VUR, and reflux nephropathy referral patterns. We analyzed trends in VUR diagnosis in subspecialty clinics (Nephrology/ Urology) from 2012 to 2015 in two major pediatric tertiary care centers.

\section{MATERIALS AND METHODS}

Numbers of new and follow-up outpatient visits were extracted from electronic medical records for years 2012-2015 with primary and/or secondary diagnoses ICD-9/10 codes (593.70/ N13.70, 593.71/N13.71, 593.72/N13.721/N13.722, and 593.73/ N13.729) to correspond with VUR, VUR unilateral, VUR bilateral, and VUR with reflux nephropathy. Data were also collected for new visits for ICD-9/10 codes 590.10/N10 and 599.0/N39 to correspond to acute pyelonephritis and UTI, respectively. Data were collected from Le Bonheur Children's Hospital (LBCH) in Memphis, TN, and Nationwide Children's Hospital (NCH) in Columbus, $\mathrm{OH}$. New cases were defined as first time visits for VUR diagnosis in either Urology or Nephrology clinics. Established cases were defined as those presenting for follow-up with previous visits in either clinic. Each patient was counted only once per year even if he/she had multiple follow-up visits that year. Data were collected for patients $0-18$ years of age. Yearto-year and average percent change over the study period were calculated and graphs generated on IBM SPSS statistics 24. Linear regression analysis was done on SAS version 9.4.

\section{RESULTS}

Annual numbers of patients visits for new and established cases of VUR and VUR nephropathy (Figure 1) and new patient referrals for UTI (Figure 2) from years 2012 to 2015 at both centers are presented. Annually, LBCH experienced an average 13\% decrease in new and established cases during the study period. A comparable pediatric center from the Midwest, $\mathrm{NCH}$, had an annual average decrease of $17 \%$ in new and established cases of VUR. From 2012 to 2015, LBCH and $\mathrm{NCH}$ experienced an average annual decrease of 22 and $27 \%$ in new and established VUR nephropathy
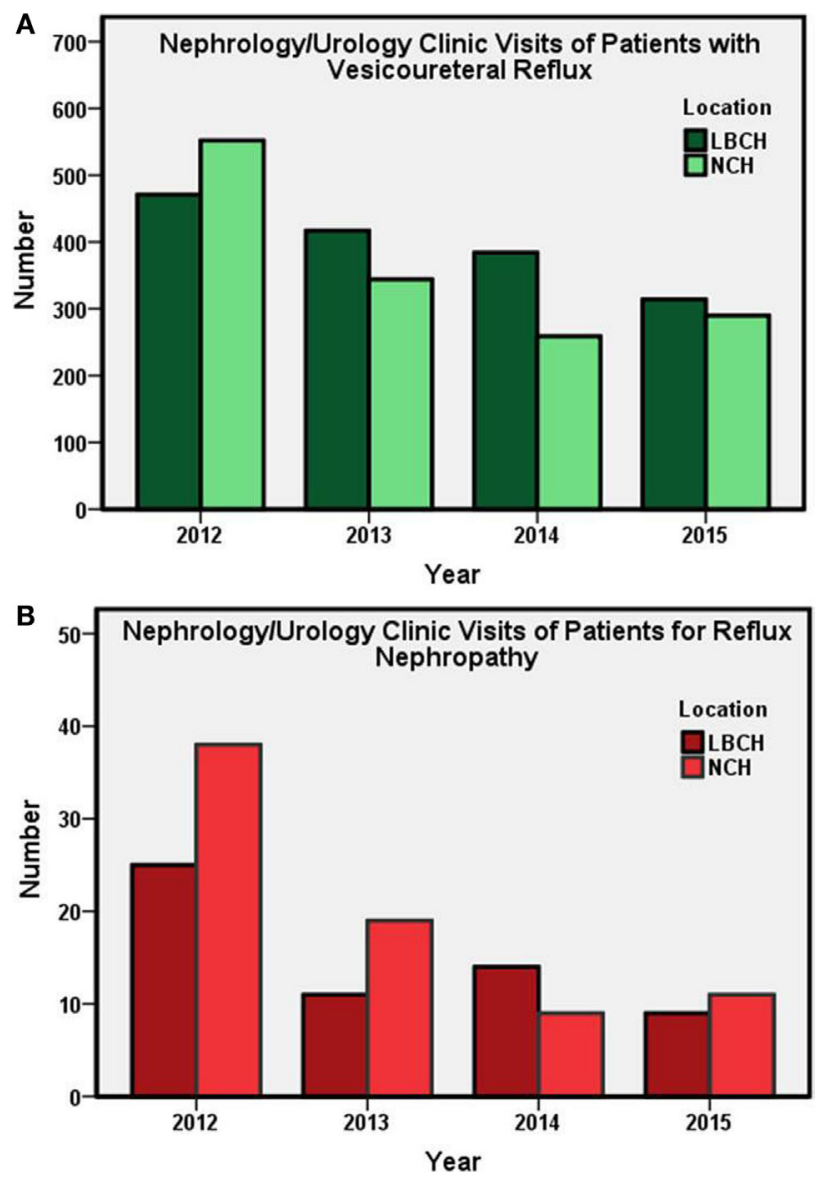

FIGURE 1 | Subspecialty (Nephrology and Urology) clinic visits (total annual number) at LBCH and NCH from 2012 to 2015. (A) Annual subspecialty clinic visits of new and established patients with VUR. (B) Number of subspecialty clinic visits for patients with VUR nephropathy. LBCH, Le Bonheur Children's Hospital; NCH, Nationwide Children's Hospital.

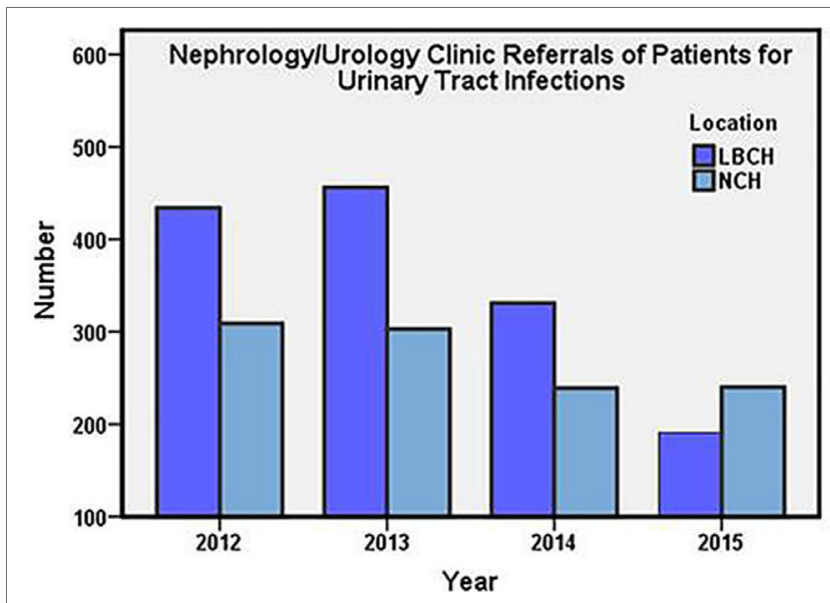

FIGURE 2 | Subspecialty (Nephrology and Urology) clinic referrals (total annual number) of new patients at $\mathrm{LBCH}$ and NCH from 2012 to 2015. LBCH, Le Bonheur Children's Hospital; NCH, Nationwide Children's Hospital. 
cases, respectively. Visits for new cases of UTI averaged an annual decrease of $16 \%$ in both centers from 2012 to 2015 . Linear regression demonstrated that number of patients (patients/year $\pm \mathrm{SE}$ ) went down $69 \pm 19(P=0.02), 7 \pm 2(P=0.02)$, and $67 \pm 25$ $(P=0.04)$ for VUR, VUR nephropathy, and UTI, respectively.

\section{DISCUSSION}

The AAP guidelines in 2011 were written with the objectives to diagnose and treat UTI in febrile children between 2 and 24 months of age and to initially screen for underlying renal anomalies by utilizing non-invasive diagnostic tests like RBUS avoiding more invasive diagnostic procedures like VCUG which require bladder catheterization and radiation exposure. The guidelines recommend using RBUS in children between 2 and 24 months of age after first febrile UTI and VCUG after the second febrile UTI. No specific guidelines exist for children older than 2 years of age. Our data suggest that the number of VCUGs performed following febrile UTIs may be decreasing leading to the decrease in VUR diagnoses. This reduced number of VCUGs may be a result of adherence to the 2011 AAP guidelines and supports other data recently published (10). Because, in our judgment, adherence to AAP guidelines would be optimized when children have close follow-up with a single primary care provider. Decreased VUR recognition may be concentrated in patients that are seen in multiple settings (Urgent care, Emergency department, and different primary care providers), thereby making application of the guidelines difficult. A third possibility is that VUR is increasingly being managed in the primary care setting, which is also supported by our finding of decreasing trend in the UTI visits at both centers. After 5 years since these guidelines were released, no new evidence has emerged indicating an ideal treatment to prevent renal scarring in patients with VUR although there is some evidence for use of CAP in preventing recurrent UTIs (6).

Our data show reduction in the cases of VUR nephropathy. From a nephrologist's point of view, an unwanted consequence of decreased VUR surveillance would also be decreased surveillance of sequelae such as renal scarring. We have identified several potential explanations for this aforementioned trend including (1) decreased diagnosis of VUR and/or VUR nephropathy due to decreased surveillance, (2) misinterpretation or incorrect application of VUR guidelines resulting in less VUR nephropathy diagnosis and management, (3) decrease in VUR nephropathy occurrence in the general population, which is very unlikely, and/ or (4) increased management of VUR nephropathy in the primary care setting, which could be in view of previous studies showing no evidence of medical or surgical management changing the progression of VUR nephropathy and even questioning the role of VUR in nephropathy $(11,12)$. On the other hand, evidence does suggest that the number of UTIs is associated with increased risk of scarring especially in girls with $\operatorname{VUR}(3,13)$.

In order to diagnose reflux nephropathy, a patient must have radiologic evidence of parenchymal abnormalities consistent with renal scarring. Additionally, new onset hypertension and/or CKD in the context of VUR would suggest reflux nephropathy and could result in ICD-9/10 coding, accordingly. We acknowledge that the lack of availability of DMSA may have resulted in decreased recognition, but our centers have employed additional imaging techniques to monitor/diagnose renal parenchymal abnormalities when clinically appropriate. While we cannot specifically analyze each case presented for the rationale for giving a diagnosis of reflux nephropathy, we cannot attribute our trends to changing clinical criteria for diagnosing VUR nephropathy. The downward trend in VUR nephropathy diagnoses is the most concerning finding in our study. While we acknowledge that this data is based on ICD9/10 coding and has inherent limitations, we cannot exclude the possibility that the trend is real. Furthermore, treatment strategies have not changed over the study period to explain a true decrease in VUR nephropathy. However, RBUS has a relatively low sensitivity for VUR and VUR nephropathy, and results of RBUS are also operator and patient dependent. A recent study showed the sensitivity and negative predictive values of RBUS for Grades I-V VUR were 52.3 and 75.1\%, and for Grades III-V VUR were 68.4 and $87.8 \%$, respectively (14). Two studies showed poor correlation between RBUS and VCUG $(15,16)$. RBUS also has poor sensitivity to detect renal scars (17). While influenced by grade of VUR, scarring risk is not isolated to only high-grade VUR (18). Thus, ignoring low-grade VUR could lead to missed VUR nephropathy recognition and support the trends we present. Our data show an upward trend in the 2015 cases that could have resulted from the transition from ICD-9 to ICD-10 coding. ICD-10-CM provides better capture of diagnoses that are missed by ICD-9-CM, and extensive training and courses on ICD-10-CM may have had effect on reporting more efficiently in 2015 (19).

\section{Limitations}

These study data are based on ICD-9/10 coding and have inherent limitations that depend upon correct code entry in the system. This small study is based upon findings of two large centers and trends at other institutions in North America are needed.

\section{CONCLUSION}

While the clinical approach to VUR has not changed to result in actual decreased incidence of disease, we conclude that the decreased number of VUR and VUR nephropathy cases identified in subspecialty clinics at two major children's hospital reflect a decreased referral for UTI and VUR to specialty clinics by primary care physicians. Based on AAP guidelines, the drop in the diagnosis of VUR may reflect reduced identification of minor cases that do not have long-term ramifications for kidney health. On the other hand, the data may reflect missing cases of VUR and nephropathy in the community that will be seen in future in trends in the causes of pediatric end-stage renal disease. We suggest that clinicians following the AAP guidelines ensure that all UTI are accounted for and surveillance is appropriately escalated for recurrent UTI or abnormal imaging results. We recommend counseling parents about the symptoms of UTI such as fever, dysuria, red color urine, and abdominal or flank pain and advising parents to seek medical advice in that situation. Moreover, if parents seek medical care for their child outside of the medical home, they should update the primary care physician about any new episodes of UTI. Thus, clinical surveillance can be appropriately escalated and appropriate imaging obtained and/ 
or subspecialty referrals can be made. Given that we only present data from two medical centers, and factors like grade of VUR and evidence of nephropathy could not be specified in each case due to the nature of study, future prospective, multicenter studies are needed to investigate these trends.

\section{ETHICS STATEMENT}

This study is from de-identified data pulled from electronic medical record database. No IRB/informed consent was necessary for data collection.

\section{REFERENCES}

1. Shaikh N, Morone NE, Bost JE, Farrell MH. Prevalence of urinary tract infection in childhood: a meta-analysis. Pediatr Infect Dis J (2008) 27:302-8. doi:10.1097/INF.0b013e31815e4122

2. Hoberman A, Charron M, Hickey RW, Baskin M, Kearney DH, Wald ER. Imaging studies after a first febrile urinary tract infection in young children. N Engl J Med (2003) 348:195-202. doi:10.1056/NEJMoa021698

3. Downs SM. Technical report: urinary tract infections in febrile infants and young children. The Urinary Tract Subcommittee of the American Academy of Pediatrics Committee on Quality Improvement. Pediatrics (1999) 103:e54. doi:10.1542/peds.103.4.e54

4. Finnell SM, Carroll AE, Downs SM; Subcommittee on Urinary Tract. Technical report-diagnosis and management of an initial UTI in febrile infants and young children. Pediatrics (2011) 128:e749-70. doi:10.1542/peds. 2011-1332

5. Pennesi M, Travan L, Peratoner L, Bordugo A, Cattaneo A, Ronfani L, et al. Is antibiotic prophylaxis in children with vesicoureteral reflux effective in preventing pyelonephritis and renal scars? A randomized, controlled trial. Pediatrics (2008) 121:e1489-94. doi:10.1542/peds.20072652

6. Investigators RT, Hoberman A, Greenfield SP, Mattoo TK, Keren R, Mathews R, et al. Antimicrobial prophylaxis for children with vesicoureteral reflux. N Engl J Med (2014) 370:2367-76. doi:10.1056/NEJMoa1401811

7. Brandstrom P, Esbjorner E, Herthelius M, Swerkersson S, Jodal U, Hansson S. The Swedish reflux trial in children: III. Urinary tract infection pattern. J Urol (2010) 184:286-91. doi:10.1016/j.juro.2010.01.061

8. Mattoo TK, Chesney RW, Greenfield SP, Hoberman A, Keren R, Mathews $\mathrm{R}$, et al. Renal scarring in the randomized intervention for children with vesicoureteral reflux (RIVUR) trial. Clin JAm Soc Nephrol (2016) 11:54-61. doi:10.2215/CJN.05210515

9. Orellana P, Baquedano P, Rangarajan V, Zhao JH, Eng ND, Fettich J, et al. Relationship between acute pyelonephritis, renal scarring, and vesicoureteral reflux. Results of a coordinated research project. Pediatr Nephrol (2004) 19:1122-6. doi:10.1007/s00467-004-1501-5

10. Jerardi KE, Elkeeb D, Weiser J, Brinkman WB. Rapid implementation of evidence-based guidelines for imaging after first urinary tract infection. Pediatrics (2013) 132:e749-55. doi:10.1542/peds.2013-0720

\section{AUTHOR CONTRIBUTIONS}

$\mathrm{AQ}, \mathrm{AS}$, and $\mathrm{DH}$ provided the concept and structure for the study. All authors involved in data analysis and interpretation. OA extracted the data. AS prepared the first draft of manuscript. AS and DH reviewed and revised the manuscript. All authors approved the final version of manuscript.

\section{FUNDING}

DH and AS were supported by R01DK106286 from NIDDK.

11. Craig JC, Irwig LM, Knight JF, Roy LP. Does treatment of vesicoureteric reflux in childhood prevent end-stage renal disease attributable to reflux nephropathy? Pediatrics (2000) 105:1236-41. doi:10.1542/peds.105.6.1236

12. Ishikura K, Uemura O, Hamasaki Y, Nakai H, Ito S, Harada R, et al. Insignificant impact of VUR on the progression of CKD in children with CAKUT. Pediatr Nephrol (2016) 31:105-12. doi:10.1007/s00467-015-3196-1

13. Swerkersson S, Jodal U, Sixt R, Stokland E, Hansson S. Relationship among vesicoureteral reflux, urinary tract infection and renal damage in children. J Urol (2007) 178:647-50. doi:10.1016/j.juro.2007.04.004

14. Hung TW, Tsai JD, Liao PF, Sheu JN. Role of renal ultrasonography in predicting vesicoureteral reflux and renal scarring in children hospitalized with a first febrile urinary tract infection. Pediatr Neonatol (2016) 57:113-9. doi:10.1016/j.pedneo.2015.06.001

15. Kurtz MP, Chow JS, Johnson EK, Rosoklija I, Logvinenko T, Nelson CP. Imaging after urinary tract infection in older children and adolescents. J Urol (2015) 193:1778-82. doi:10.1016/j.juro.2014.10.119

16. Logvinenko T, Chow JS, Nelson CP. Predictive value of specific ultrasound findings when used as a screening test for abnormalities on VCUG. J Pediatr Urol (2015) 11:176.e1-7. doi:10.1016/j.jpurol.2015.03.006

17. Moorthy I, Wheat D, Gordon I. Ultrasonography in the evaluation of renal scarring using DMSA scan as the gold standard. Pediatr Nephrol (2004) 19:153-6. doi:10.1007/s00467-003-1363-2

18. Keren R, Shaikh N, Pohl H, Gravens-Mueller L, Ivanova A, Zaoutis L, et al. Risk factors for recurrent urinary tract infection and renal scarring. Pediatrics (2015) 136:e13-21. doi:10.1542/peds.2015-0409

19. Watzlaf V, Alkarwi Z, Meyers S, Sheridan P. Physicians' outlook on ICD-10-CM/PCS and its effect on their practice. Perspect Health Inf Manag (2015) $12: 1 b$.

Conflict of Interest Statement: AS has consulted for Allena Pharmaceuticals. All other authors have no conflict of interest.

Copyright (c) 2017 Qureshi, Ajayi, Schwaderer and Hains. This is an open-access article distributed under the terms of the Creative Commons Attribution License (CC $B Y)$. The use, distribution or reproduction in other forums is permitted, provided the original author(s) or licensor are credited and that the original publication in this journal is cited, in accordance with accepted academic practice. No use, distribution or reproduction is permitted which does not comply with these terms. 